\title{
Systematic and random errors in rotating-analyzer ellipsometry
}

\author{
J. M. M. de Nijs and A. van Silfhout \\ Faculty of Applied Physics, Twente University, P.O. Box 217, 7500 AE Enschende, The Netherlands
}

Received August 14, 1987; accepted January 26, 1988

\begin{abstract}
Errors and error sources occurring in rotating-analyzer ellipsometry are discussed. From general considerations it is shown that a rotating-analyzer ellipsometer is inaccurate if applied at $P=0^{\circ}$ and in cases when $\psi=0^{\circ}$ or where $\Delta$ is near $0^{\circ}$ or $180^{\circ}$. Window errors, component imperfections, azimuth errors and all other errors may, to first order, be treated independently and can subsequently be added. Explicit first-order expressions for the errors $\delta \Delta$ and $\delta \psi$ caused by windows, component imperfections, and azimuth errors are derived, showing that all of them, except the window errors, are eliminated in a two-zone measurement. Higher-order errors that are due to azimuth errors are studied numerically, revealing that they are in general less than $0.1^{\circ}$. Statistical errors are also discussed. Errors caused by noise and by correlated perturbations, i.e., periodic fluctuations of the light source, are also considered. Such periodic perturbations do cause random errors, especially when they have frequencies near $2 \omega_{A}$ and $4 \omega_{A}$.
\end{abstract}

\section{INTRODUCTION}

During the past decade automated ellipsometers have in many cases superseded the traditional nulling ellipsometers. These nulling ellipsometers are based on the so-called totalextinction principle; $\Delta$ and $\psi$ are obtained from the component readings when the transmitted beam is totally extinguished. For a number of reasons, errors do occur in these measurements; these errors are extensively discussed by Azzam and Bashara, ${ }^{1-4}$ Aspens, ${ }^{5}$ Straaijer et al., ${ }^{6}$ and McCrackin. ${ }^{7}$ The automation of the ellipsometer has resulted in a number of different types, 8,9 of which the rotating-analyzer ellipsometer ${ }^{10,11}$ (RAE) is the most important. With this automation the total-extinction method has been replaced by a total-intensity measurement. In the case of the RAE the light flux as a function of the analyzer angle is measured: $I_{\text {det }}=I_{\text {det }}(A)$. From this intensity the two desired quantities, $\Delta$ and $\psi$, are calculated.

With the introduction of the automated ellipsometers of the RAE type, the error analysis had to be repeated. Azzam and Bashara ${ }^{12}$ presented such a study, showing that a twozone measurement, as known for the nulling ellipsometer, existed, but they did not present explicit expressions for $\delta \Delta$ and $\delta \psi$ as they did for the nulling ellipsometer. ${ }^{2}$ Such explicit expressions should be of great value for deciding whether a two-zone measurement is required or whether a single-zone measurement suffices. Aspnes ${ }^{13}$ discussed the effect of noise in the RAE; noise introduces statistical errors in $\Delta$ and $\psi$. However, other sources besides noise contribute to statistical errors, as will be shown in this paper. Finally, we should mention another paper of Aspnes, ${ }^{14}$ which is perhaps a little outside the scope of this paper, on calibration errors caused by imperfect polarizers.

All references mentioned concern first-order errors. The effect of each error source is studied individually; the remaining part of the ellipsometer is assumed to be ideal. Subsequently, all errors attributed to the different sources are added up. In this paper we apply the same principle in our derivation of simple expressions for first-order errors in
$\Delta$ and $\psi$ obtained by a RAE. Most of these errors appear to be antisymmetric and consequently can be eliminated by a two-zone measurement. When first-order errors are eliminated in this way, the question arises about when secondorder errors, particularly those caused by azimuth errors, can no longer be neglected. This problem cannot be solved analytically and has to be approached numerically. For this purpose we wrote a computer program that simulates the optical part of the RAE. Subsequently we simulated the usual calibrations and measurements. The errors in $\Delta$ and $\psi, \delta \Delta$, and $\delta \psi$ then were obtained from the difference between the $\Delta$ and $\psi$ values introduced in the simulation part and those resulting from the measurement.

In Section 2 we present a short introduction to the RAE. Subsequently some considerations on general error propagation are discussed in Section 3, from which one arrives at the conclusion that a RAE yields good results unless $P, \Delta$, or $\psi$ approaches 0 or $\pi$. Section 4 contains an introduction of the optics and our approach to the present problem, which is based on the idea of Taylor expansions. In combination with the result of Section 2, it gives us a formalism that allows us to calculate, to first order, the errors $\delta \Delta$ and $\delta \psi$ caused by any source. Section 5 proceeds with the application of this formalism to systematic errors, such as azimuth errors, component imperfection, and windows. Apart from these systematic errors, we also consider statistical errors. This subject is discussed extensively in Section 6. Finally, we conclude with a discussion of the currently obtained results.

\section{THE ROTATING-ANALYZER ELLIPSOMETER}

Figure 1 presents a diagram of a RAE. The optical system contains a polarizer, a sample, a rotating analyzer, and, optionally, a compensator. A detailed description of a RAE can be found in Ref. 10. The ideal polarizer and analyzer are completely characterized by the azimuth angles $P$ and $A$, 


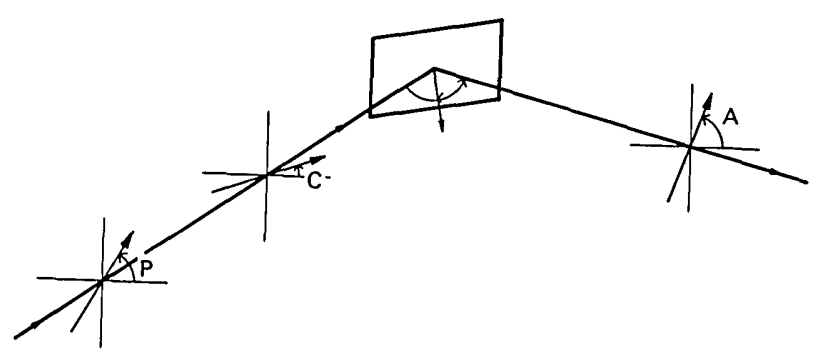

Fig. 1. Schematic diagram of an ideal ellipsometer. The optical part of the system consists of a polarizer, an optional compensator, the sample, and an analyzer. In the case of a RAE the analyzer rotates with constant angular velocity. The angles $P, C$, and $A$ are the angles of the characteristic axes of the components measured relative to the plane of incidence.

which their transmission axes make with the plane of incidence. The compensator fast-axis azimuth from the plane of incidence is denoted by $C$, and its phase retardation is denoted by $Q$. In the ideal situation the compensator is assumed to be positioned with its fast axis aligned with the plane of incidence $(C=0) .{ }^{13}$ This configuration makes the phase retardations of the sample $(\Delta)$ and of the compensator directly additive, and $\psi$ remains unchanged. ${ }^{12}$ One may as well omit the presence of the compensator if the sample is characterized by $\Delta^{\prime}=\Delta+Q, \psi^{\prime}=\psi$. For this ideal situation the light flux transmitted by the rotating analyzer $\left(A=\omega_{A} t\right)$ becomes

$$
\begin{aligned}
I= & I_{0}\left[1-\mathrm{C}_{2 P} \mathrm{C}_{2 \psi}+\left(\mathrm{C}_{2 P}-\mathrm{C}_{2 \psi}\right) \cos 2 \omega_{A} t\right. \\
& \left.+\mathrm{S}_{2 P} \mathrm{~S}_{2 \psi} \mathrm{C}_{\Delta} \sin 2 \omega_{A} t\right],
\end{aligned}
$$

where $\mathrm{S}$ and $\mathrm{C}$ are shorthand notation for sine and cosine, respectively.

The light flux as a function of time, $I_{\operatorname{det}}(t)$, transmitted by the analyzer is the actually measured quantity, having the mathematical form

$$
I_{\mathrm{det}}(t)=\alpha_{0}+\alpha_{c} \cos 2 \omega_{A} t+\alpha_{s} \sin 2 \omega_{A} t .
$$

In measurement the coefficient $\alpha_{0}$ is obtained from $I_{\text {det }}(t)$ by averaging $I_{\text {det }}$ over a number of analyzer cycles:

$$
\alpha_{0}=\left\langle I_{\text {det }}\right\rangle \text {, }
$$

while $\alpha_{s}$ and $\alpha_{c}$ are retrieved by the Fourier integral

$$
\alpha_{c}+i \alpha_{s}=\frac{2}{\tau} \int_{0}^{\tau} I_{\operatorname{det}}(t) \exp \left(i 2 \omega_{A} t\right) \mathrm{d} t, \quad \tau=\frac{4 \pi}{\omega_{A}} N,
$$

The preceding discussion is correct only for the ideal RAE; in practice things appear less ideal than assumed. One should realize that the assumption of Eq. (2) on the detected intensity is not realistic. Noise and intensity modulations other than the $2 \omega_{A}$ component will be present, causing random errors. In Section 6 we present a quantitative treatment of this subject. Apart from these random errors, a number of systematic errors, hence less-noticeable errors, do enter the measurement. The polarizers used are not perfect, they transmit slightly elliptically polarized light instead of perfect linearly polarized light. In a number of cases, for example, if the sample is situated inside an ultrahigh-vacuum system, windows are used, thus distorting the state of polarization of the light beam. The plane of incidence is assumed to be known exactly, and, if this is not the case, azimuth errors in $P, C$, and $A$ are introduced, ultimately causing systematic errors in $\Delta$ and $\psi$.

\section{GENERAL ERROR PROPAGATION}

The accuracy with which $\Delta$ and $\psi$ can be determined strongly depends on the nature of the sample. To study this feature and for later purposes, we first consider the propagation of errors in general.

The light flux detected contains, in principle, a dc and a $2 \omega_{A}$ component only, which are retrieved by averaging and by Fourier analysis. Consequently all errors independent of their origin propagate only through the coefficients $\alpha_{0}, \alpha_{c}$, and $\alpha_{s}$. It is for this reason mainly that we are interested in what errors $\delta \Delta$ and $\delta \psi$ are introduced by the shifts $\delta \alpha_{0}, \delta \alpha_{c}$, and $\delta \alpha_{s}$.

From Eqs. (4a) and (4b) we can derive the Jacobian matrix, relating the errors in $\Delta$ and $\psi$ to those in the normalized Fourier coefficients $\hat{\alpha}_{\mathrm{c}}=\alpha_{c} / \alpha_{0}$ and $\hat{\alpha}_{s}=\alpha_{s} / \alpha_{0}$

$\left[\begin{array}{l}\delta \Delta \\ \delta \psi\end{array}\right]=\frac{1-\mathrm{C}_{2 P} \mathrm{C}_{2 \psi}}{\mathrm{S}_{2 P}{ }^{2} \mathrm{~S}_{2 \psi}{ }^{2} \mathrm{~S}_{\Delta}}\left[\begin{array}{cc}\left(\mathrm{C}_{2 \psi}-\mathrm{C}_{2 P}\right) \mathrm{C}_{\Delta} & -\mathrm{S}_{2 P} \mathrm{~S}_{2 \psi} \\ 1 / 2\left(1-\mathrm{C}_{2 P} \mathrm{C}_{2 \psi}\right) \mathrm{S}_{2 \psi} \mathrm{S}_{\Delta} & 0\end{array}\right]\left[\begin{array}{l}\delta \hat{\alpha}_{c} \\ \delta \hat{\alpha}_{s}\end{array}\right]$.

A look at the denominator of the expression in front of the matrix unambiguously shows that the RAE is insensitive if used at small $P$ or if applied to a sample with $\mathrm{S}_{\Delta} \approx 0$ or small $\psi$. Under these conditions, even small errors $\delta \hat{\alpha}_{c}$ and $\delta \hat{\alpha}_{s}$ do cause large errors in $\Delta$ and $\psi$. It is for this reason that a compensator is advised in cases of $\Delta$ near $0, \pi$ rad. ${ }^{13}$

Another Jacobian matrix relating $\delta \hat{\alpha}_{c}$ and $\delta \hat{\alpha}_{s}$ to $\delta \alpha_{0}, \delta \alpha_{c}$, and $\delta \alpha_{s}$ eliminates $\delta \hat{\alpha}_{c}$ and $\delta \hat{\alpha}_{s}$ from Eq. (5), thus yielding

$$
\left[\begin{array}{l}
\delta \Delta \\
\delta \psi
\end{array}\right]=\frac{1}{\mathrm{~S}_{2 P}{ }^{2} \mathrm{~S}_{2 \psi}{ }^{2} \mathrm{~S}_{\Delta}}\left[\begin{array}{ccc}
\left(1-\mathrm{C}_{2 P} \mathrm{C}_{2 \psi}\right) \mathrm{C}_{\Delta} & \left(\mathrm{C}_{2 \psi}-\mathrm{C}_{2 P}\right) \mathrm{C}_{\Delta} & -\mathrm{S}_{2 P} \mathrm{~S}_{2 \psi} \\
1 / 2\left(\mathrm{C}_{2 \psi}-\mathrm{C}_{2 P}\right) \mathrm{S}_{2 \psi} \mathrm{S}_{\Delta} & 1 / 2\left(1-\mathrm{C}_{2 P} \mathrm{C}_{2 \psi}\right) \mathrm{S}_{2 \psi} \mathrm{S}_{\Delta} & 0
\end{array}\right]\left[\begin{array}{l}
\delta \alpha_{0} \\
\delta \alpha_{c} \\
\delta \alpha_{s}
\end{array}\right],
$$

where $N$ is the number of analyzer cycles.

Thus, having determined the coefficients $\alpha_{0}, \alpha_{c}$, and $\alpha_{s}$ from the measured light flux $I_{\operatorname{det}}$, we find $\Delta$ and $\psi$ by equating Eqs. (1) and (2):

$$
\begin{gathered}
\frac{\mathrm{C}_{2 P}-\mathrm{C}_{2 \psi}}{1-\mathrm{C}_{2 P} \mathrm{C}_{2 \psi}}=\frac{\alpha_{c}}{\alpha_{0}}, \\
\frac{\mathrm{S}_{2 P} \mathrm{~S}_{2 \psi} \mathrm{C}_{\Delta}}{1-\mathrm{C}_{2 P} \mathrm{C}_{2 \psi}}=\frac{\alpha_{s}}{\alpha_{0}} .
\end{gathered}
$$

which is the general first-order error-propagation matrix expressed in terms of the variables $\Delta, \psi$, and $P$.

\section{THEORETICAL FORMALISM CONCERNING ERROR CALCULATIONS}

In Section 3 we derived Eq. (6), which relates the errors in $\Delta$ and $\psi$ to those in the coefficients $\alpha_{0}, \alpha_{c}$, and $\alpha_{s}$. An azimuth error $\delta P$ of the polarizer resulting from the calibration, for example, is a source for such systematic shifts of $\alpha_{0}, \alpha_{c}$, and 
$\alpha_{s}$. These systematic errors $\delta \alpha_{0}, \delta \alpha_{c}$, and $\delta \alpha_{s}$ caused by azimuth angle errors, component imperfection, and windows can be calculated, to first-order, as is shown in this section.

The state of polarization of a monochromatic light beam can be described by a Stokes vector S. ${ }^{4}$ Together with Mueller matrices we have a formalism well fitted for our purpose; the second-order numerical calculations are easily programmable, and the analytical manipulations are straightforward. In the present treatment we distinguish the transmission matrix of a component in its own frame of reference $(\mathcal{T})$ and the rotation matrix $[R(\theta)]$ performing the transformation of $\mathbf{S}$ from one frame of reference to another, rotated over an angle $\theta$. Concrete expressions for $\mathcal{R}$ and $\mathcal{T}$ can be found in the papers of Azzam and Bashara. ${ }^{1,4}$ In this way the response matrix of a component $(k)$ in an arbitrary frame of reference becomes $\mathcal{R}^{-1} \mathcal{T}_{k} \mathcal{R}$. Denoting the Stokes vector describing the light beam incident upon the ideal optical system by $\mathbf{S}_{i}{ }^{\circ}$, we obtain for the Stokes vector for transmitted light beam $\left(\mathbf{S}_{f}{ }^{\circ}\right)$ the equation

$$
\mathbf{S}_{f}^{\circ}=\mathcal{T}_{A}^{\circ} \cdot \mathscr{R}\left(A^{\circ}\right) \mathcal{T}_{S}{ }^{\circ} \mathcal{T}_{C}{ }^{\circ} \mathcal{R}^{-1}\left(P^{\circ}\right) \mathcal{T}_{P}{ }^{\circ} \mathbf{S}_{i}{ }^{\circ},
$$

where $\mathcal{T}_{S}$ and $\mathcal{T}_{C}$ are the Mueller matrices of the sample and the compensator. All variables marked ${ }^{\circ}$ indicate that we consider the ideal components for the moment. Furthermore, we have suppressed the two matrices $\mathcal{R}\left(P^{\circ}\right)$ and $R^{-1}\left(A^{\circ}\right)$ because it does not matter in which frame of reference $\mathbf{S}_{i}$ and $\mathbf{S}_{f}$ are represented. Reminding ourselves that the ellipsometer is not ideal, we introduce all real azimuth angles, the entrance and exit windows, which are, respectively, denoted by $\mathcal{T}_{w}$ and $\mathcal{T}_{w^{\prime}}$, and the imperfect components. Then $\mathbf{S}_{f}$ becomes

$$
\mathbf{S}_{f}=\mathcal{T}_{A} \mathcal{R}(A) \mathcal{T}_{w^{\prime}} \mathcal{T}_{S} \mathcal{T}_{w} \mathcal{R}^{-1}(C) \mathcal{T}_{C} \mathscr{R}(C) \mathcal{R}^{-1}(P) \mathcal{T}_{P} \mathbf{S}_{i}
$$

The real azimuth angles $\theta$ are related to the ideal ones by $\theta=$ $\theta^{\circ}+\delta \theta$. Introducing $\theta^{\circ}+\delta \theta$ in the rotation matrix $\mathcal{R}$, we can make a Taylor expansion of $\mathcal{R}$ in the variable $\delta \theta$ :

$$
\mathscr{R}(\theta)=\mathscr{R}\left(\theta^{\circ}\right)+\frac{\mathrm{d} \mathscr{R}}{\mathrm{d} \theta} \delta \theta+\frac{1}{2} \frac{\mathrm{d}^{2} \mathcal{R}}{\mathrm{d} \theta^{2}} \delta \theta^{2}+\ldots
$$

The transmission matrices also can be expanded if we use a variable $\xi$ to describe the imperfection; for example, $\xi$ can represent the extinction coefficient of the nonideal polarizer:

$$
\mathcal{T}(\xi)=\mathcal{T}^{\circ}+\frac{\mathrm{d} \mathcal{T}}{\mathrm{d} \xi} \delta \xi+\frac{1}{2} \frac{\mathrm{d}^{2} \mathcal{T}}{\mathrm{d} \xi^{2}} \delta \xi^{2}+\ldots
$$

If we now substitute Eqs. (9) and (10) into Eq. (8), we obtain, for $\mathbf{S}_{f}$,

$$
\mathbf{S}_{f}=\mathbf{S}_{f}{ }^{\circ}+\sum_{k} \delta \mathbf{S}_{f}^{k}+\sum_{\substack{k, l \\ k \neq l}} \delta \mathbf{S}_{f}^{k, l}+\sum_{k} \delta \mathbf{S}_{f}^{k, k}+\ldots
$$

where $k$ and $l$ correspond to the individual error sources. Thus $\delta \mathbf{S}_{f}^{k}$ is the perturbation caused solely by the first-order imperfection of the $k$ th component; the remaining optical system is assumed to be ideal. Similarly, $\delta \mathbf{S}_{f}^{k l}$ denotes the extra distortion due to the perturbing action of the firstorder imperfection of the $l$ th component on the first-order perturbation of the light beam already caused by the $k$ th component. $\delta \mathbf{S}_{f}^{k k}$ is the distortion to be attributed to the second-order effects of the $k$ th component. The second term on the right-hand side of Eq. (11) is the linear one, which we shall treat mainly. The nonlinear third and fourth terms are negligible if the imperfections are small.

In this way we have arrived at a linear approximation of error propagation in the RAE:

$$
\begin{aligned}
\mathbf{S}_{f} \approx & \mathbf{S}_{f}{ }^{\circ}+\sum_{k} \delta \mathbf{S}_{f}{ }^{k}=\mathbf{S}_{f}^{\circ} \\
& +\left[\mathcal{T}_{A}^{\circ} \mathcal{R}\left(A^{\circ}\right) \mathcal{T}_{S}^{\circ} \mathcal{T}_{C}^{\circ} \mathcal{R}^{-1}\left(P^{\circ}\right) \frac{\mathrm{d} \mathcal{T}_{P}}{\mathrm{~d} \gamma_{P}} \delta \gamma_{P}\right. \\
& +\mathcal{T}_{A}^{\circ} \mathcal{R}\left(A^{\circ}\right) \mathcal{T}_{S}^{\circ} \mathcal{T}_{C}^{\circ} \frac{\mathrm{d} \mathcal{R}^{-1}}{\mathrm{~d} P} \delta P \mathcal{T}_{P}^{\circ} \\
& \left.+\ldots+\frac{\mathrm{d} \mathcal{T}_{A}}{\mathrm{~d} \gamma_{A}} \delta \gamma_{A} \mathcal{R}\left(A^{\circ}\right) \mathcal{T}_{S}{ }^{\circ} \mathcal{T}_{C}{ }^{\circ} \mathcal{R}^{-1}\left(P^{\circ}\right) \mathcal{T}_{P}{ }^{\circ}\right] \mathbf{S}_{i}^{\circ},
\end{aligned}
$$

where we have suppressed $\mathcal{T}_{w^{\prime}}{ }^{\circ}$ and $\mathcal{T}_{w}{ }^{\circ}$ because $\mathcal{T}_{w^{\prime}}{ }^{\circ}=\mathcal{T}_{w}{ }^{\circ}$ $=1$. Using this formalism, we can calculate every perturbation $\delta \mathbf{S}_{f}^{k}$ of the Stokes vector $\mathbf{S}_{f}$. However, our interest is in the coefficients $\alpha_{0}, \alpha_{c}$, and $\alpha_{s}$. For all error sources except those attributed to the analyzer, we simply note that the intensity $\left[\mathbf{S}_{f}\right]_{0}$ is the detected quantity; multiplying $\mathbf{S}_{f}$ by $\delta_{1}$ $=[1,0,0,0]$ on the left-hand side yields $I_{\text {det }}$ :

$$
\delta_{1} \cdot \mathbf{S}_{f}=\left(\mathbf{S}_{f}\right)_{0}=I_{\operatorname{det}}=\delta_{1} \mathcal{T}_{A}^{\circ} \cdot \mathcal{R}\left(A^{\circ}\right) \mathbf{S}_{w^{\prime}}=\left(1, \mathrm{C}_{2 A}, \mathrm{~S}_{2 A}, 0\right) \mathbf{S}_{w^{\prime}}
$$

where $\mathbf{S}_{w^{\prime}}$ corresponds with the light flux transmitted by the exit window. From Eqs. (13) and (2), we identify

$$
\mathbf{S}_{w^{\prime}}=\left[\begin{array}{c}
\alpha_{0} \\
\alpha_{c} \\
\alpha_{s} \\
-
\end{array}\right], \quad \delta \mathbf{S}_{w^{\prime}}=\left[\begin{array}{c}
\delta \alpha_{0} \\
\delta \alpha_{c} \\
\delta \alpha_{s} \\
-
\end{array}\right] .
$$

These results clearly show that a RAE measures the first three elements of the Stokes vector representing the light beam upon the analyzer. Any shift of these directly affects $\Delta$ and $\psi$ according to Eq. (6). Errors contributed by the analyzer are excepted from Eq. (13); for those errors $\delta \mathbf{S}_{f}$ has to be calculated, after which we can perform an identification similar to that which was done in obtaining Eq. (14).

It is shown in Section 5 that most errors are antisymmetric in $P$ and consequently can be eliminated by averaging two measurements performed at $P$ and $-P$. Then the third and fourth terms on the right-hand side of Eq. (11) become significant. In some cases it should be interesting to know whether second-order errors of the fourth term in Eq. (11) are negligible. We then might assume that the contributions of the third term are negligible also, and errors can be ascribed mainly to sources that do not average, i.e., windows. For this reason we shall study the fourth term of Eq. (11) the second-order effects of one component while the remaining part is assumed ideal. However, in spite of the fact that $\delta \mathbf{S}_{f}^{k, k}$ is exact, its propagation up to $\Delta$ and $\psi$ cannot be studied by using Eq. (6) because Eq. (6) is first order only. Consequently the problem has to be approached by a nu- 
merical method. Equation (8) is implemented in a numerical-simulation procedure for the optical part of the RAE. Using this procedure, we can easily obtain the perturbed $\mathbf{S}_{f}$ for the nonideal system and any particular $\Delta$ and $\psi$ that enter through $\tau_{S}{ }^{\circ}$. Subsequently Eqs. (3) and (4) are used to calculate the experimental $\Delta^{\prime}$ and $\psi^{\prime}$. The errors then are obtained from the difference between the $\Delta$ and $\psi$ entered in the simulation part and the experimental $\Delta^{\prime}$ and $\psi^{\prime}$.

\section{SYSTEMATIC ERRORS: POLARIZER IMPERFECTION, AZIMUTH ERRORS, AND WINDOWS}

In this section we discuss a number of systematic errors. In all cases we apply the same approach, outlined in the previous section. After a short introduction of a particular error we present the proper Taylor expansion. The geometrical calculations are quite tedious, although straightforward, and are left out. We do present the intermediate $\left[\delta \alpha_{0}, \delta \alpha_{c}, \delta \alpha_{s}\right.$, -] and the final $[\delta \Delta, \delta \psi]$ results, which are discussed below. In this way we treat polarizer imperfections, azimuth errors in the first- and second-order approximation, and, finally, the window imperfections. To obtain a better overview, all results of $\delta \Delta$ and $\delta \psi$ were placed in Table 1 .

\section{A. Polarizer Imperfection}

In the past some papers ${ }^{12,14}$ on polarizer imperfection have appeared. It is generally assumed that the imperfect polarizer transmits a vibration with small ellipticity. Aspnes ${ }^{14}$ used a simple Jones matrix for the imperfect polarizer; however, the transmitted beam was not represented in an orthogonal frame of reference and therefore cannot be used here. In the present case we adopt an expression for an elliptic ideal polarizer from Azzam and Bashara4:

$$
\frac{1}{1+\gamma^{2}}\left[\begin{array}{cc}
1 & -i \gamma \\
+i \gamma & \gamma^{2}
\end{array}\right],
$$

which, translated into the Mueller formalism, ${ }^{1}$ becomes

$$
\tau_{p}=1 / 2\left[\begin{array}{cccc}
1+2 \gamma^{2} & 1 & 0 & +2 \gamma \\
1 & 1-2 \gamma^{2} & 0 & +2 \gamma \\
0 & 0 & 4 \gamma^{2} & 0 \\
+2 \gamma & +2 \gamma & 0 & 0
\end{array}\right] .
$$

Retaining the coefficients of order $\gamma$ only, we get the perturbing matrix

$$
\delta \mathcal{T}_{p}=\gamma\left[\begin{array}{llll}
0 & 0 & 0 & 1 \\
0 & 0 & 0 & 1 \\
0 & 0 & 0 & 0 \\
1 & 1 & 0 & 0
\end{array}\right]
$$

Now let us first deal with the polarizer. Certainly when a monochromator or laser is used as a light source, we should not expect the light beam to be completely unpolarized or completely polarized. However, from Eq. (15) it is seen that the light beam $\left(\mathbf{S}_{P}\right)$ transmitted by the polarizer always is, to first order, slightly elliptically polarized, independent of the light source:

$$
\mathbf{S}_{P}=(1,1,0,0)+2 \gamma_{p}(0,0,0,1)+0\left(\gamma^{2}\right)
$$

Obviously we should not care too much about the light source, as long as the light flux passing the polarizer suffices. On substitution of Eq. (16) into Eq. (12) we find that

$$
\delta \mathbf{S}_{f}^{p}=\left(1, \mathrm{C}_{2 A}, \mathrm{~S}_{2 A}, 0\right) \mathcal{T}_{S}{ }^{\circ} \mathcal{T}_{C}{ }^{\circ} \mathcal{R}^{-1}\left(P^{\circ}\right) \delta \mathcal{T}_{p} \mathbf{S}_{i}^{\circ}
$$

After we make the correct identification with Eq. (14), this yields

$$
\left[\begin{array}{c}
\delta \alpha_{0} \\
\delta \alpha_{c} \\
\delta \alpha_{s} \\
-
\end{array}\right]=+2 \gamma_{P} \mathrm{~S}_{2 \psi}\left[\begin{array}{c}
0 \\
0 \\
\mathrm{~S}_{\Delta+Q} \\
\mathrm{C}_{\Delta+Q}
\end{array}\right],
$$

or, from Eq. (6), we obtain

$$
\left[\begin{array}{l}
\delta \Delta \\
\delta \psi
\end{array}\right]=-2 \gamma_{P}\left[\begin{array}{c}
1 / \mathrm{S}_{2 P} \\
0
\end{array}\right]
$$

for the systematic errors in $\Delta$ and $\psi$ caused by the imperfection $\gamma_{p}$. We can directly notice that the imperfection does not affect $\psi$. Furthermore, $\delta \Delta$ depends only on the polarizer angle $P$, not on $\Delta$ or $\psi$, and is eliminated in a two-zone measurement.

Next we consider the rotating analyzer. Substituting the imperfect Mueller matrix and performing the product $\delta_{\tau_{A}} R\left(A^{\circ}\right) \mathcal{T}_{S}{ }^{\circ} \mathcal{T}_{C}{ }^{\circ} \mathcal{R}^{-1}\left(P^{\circ}\right) \mathcal{T}_{P}{ }^{\circ} \mathbf{S}_{i}{ }^{\circ}$ results in an intensity shift of $\delta I_{\text {det }}=-2 \gamma_{A} \mathrm{~S}_{2 P} \mathrm{~S}_{2 \psi} \mathrm{S}_{\Delta+Q}$. Hence only the unmodulated component $\alpha_{0}$ is involved:

Table 1. First-Order Errors $\delta \Delta$ and $\delta \psi$ Caused by Polarizer Imperfections, Azimuth Errors, and Windows

\begin{tabular}{lll}
\hline \multicolumn{1}{c}{ Source } & \multicolumn{1}{c}{$\delta \Delta$} & \multicolumn{1}{c}{$\delta \psi$} \\
\hline Polarizer imperfection $\gamma_{P}$ & $-2 \gamma_{P} / \mathrm{S}_{2 P}$ & - \\
Analyzer imperfection $\gamma_{A}$ & $-2 \gamma_{A}\left(1-\mathrm{C}_{2 P} \mathrm{C}_{2 \psi}\right) \mathrm{C}_{\Delta+Q} / \mathrm{S}_{2 P} \mathrm{~S}_{2 \psi}$ & $\gamma_{A}\left(\mathrm{C}_{2 P}-\mathrm{C}_{2 \psi}\right) \mathrm{S}_{\Delta+Q} / \mathrm{S}_{2 P}$ \\
Azimuth errors & - & $\delta P \mathrm{~S}_{2 \psi} / \mathrm{S}_{2 P}$ \\
$\quad$ Polarizer $\delta P$ & $\delta A 2\left(\mathrm{C}_{2 P}-\mathrm{C}_{2 \psi}\right) \mathrm{S}_{\Delta+Q} / \mathrm{S}_{2 P} \mathrm{~S}_{2 \psi}$ & $\delta A\left(1-\mathrm{C}_{2 P} \mathrm{C}_{2 \psi}\right) \mathrm{C}_{\Delta+Q} / \mathrm{S}_{2 P}$ \\
Analyzer $\delta A$ & $-2 \delta C \mathrm{~S}_{Q} \mathrm{C}_{2 P} / \mathrm{S}_{2 P}$ & $\delta C\left(1-\mathrm{C}_{Q}\right) \mathrm{S}_{2 \psi} / \mathrm{S}_{2 P}$ \\
Compensator $\delta C$ & $\frac{\mathrm{S}_{2 P} \mathrm{C}_{2 \phi w}-\mathrm{C}_{Q} \mathrm{C}_{2 P} \mathrm{~S}_{2 \phi w}}{\mathrm{~S}_{2 \psi}} \delta w$ & $\frac{\mathrm{S}_{Q} \mathrm{~S}_{2 \psi} \mathrm{S}_{2 \phi w}}{2 \mathrm{~S}_{2 P}} \delta w$ \\
Windows & $\frac{\mathrm{S}_{\Delta+Q} \mathrm{~S}_{2 \phi w}{ }^{\prime}}{2 \mathrm{~S}_{2 P}}\left(1-\mathrm{C}_{2 P} \mathrm{C}_{2 \psi}\right) \delta w^{\prime}$
\end{tabular}




$$
\left[\begin{array}{c}
\delta \alpha_{0} \\
\delta \alpha_{c} \\
\delta \alpha_{s} \\
-
\end{array}\right]=-2 \gamma_{A}\left[\begin{array}{c}
\mathrm{S}_{2 P} \mathrm{~S}_{2 \psi} \mathrm{S}_{\Delta+Q} \\
0 \\
0 \\
-
\end{array}\right],
$$

which, with the help of Eq. (6), yields

$$
\left[\begin{array}{c}
\delta \Delta \\
\delta \psi
\end{array}\right]=-\frac{\gamma_{A}}{\mathrm{~S}_{2 P} \mathrm{~S}_{2 \psi}}\left[\begin{array}{c}
2\left(1-\mathrm{C}_{2 P} \mathrm{C}_{2 \psi}\right) \mathrm{C}_{\Delta+Q} \\
\left(\mathrm{C}_{2 \psi}-\mathrm{C}_{2 P}\right) \mathrm{S}_{2 \psi} \mathrm{S}_{\Delta+Q}
\end{array}\right] .
$$

Again we find expressions antisymmetric in $P$. From Eqs. (18) and (19) it follows that these errors can be eliminated, to first order, if we perform a two-zone measurement. This procedure is strongly advised with respect to absolute ellipsometry or differential ellipsometry in cases when $\Delta$ and $\psi$ change much because even good polarizers (i.e., GlanThompson) exhibit extinction coefficients of $10^{-3}$ or greater, causing errors of the order of $1^{\circ}$.

\section{B. Azimuth Errors}

Azimuth errors are introduced mainly by the calibration. Aspnes ${ }^{13}$ already showed that imperfect polarizers disturb the determination of the plane of incidence, and the same may be expected from the windows. Once an offset has entered the location of the plane of incidence, it shows up in all angle settings of $P^{\circ}, A^{\circ}$, and $C^{\circ}$, thus causing a number of systematic errors. From Eq. (9) the perturbation matrix $\delta R(\theta)$ becomes

$$
\delta \mathscr{R}(\theta)=\left[\begin{array}{cccc}
0 & 0 & 0 & 0 \\
0 & -\sin \theta & \cos \theta & 0 \\
0 & -\cos \theta & -\sin \theta & 0 \\
0 & 0 & 0 & 0
\end{array}\right] .
$$

First we discuss the polarizer azimuth error $\delta P$. Using Eqs. (12) and (14), we find that it causes the perturbation

$$
\left[\begin{array}{c}
\delta \alpha_{0} \\
\delta \alpha_{c} \\
\delta \alpha_{s} \\
-
\end{array}\right]=\mathcal{T}_{S}{ }^{\circ} \mathcal{T}_{C}{ }^{\circ} \delta \mathcal{R}(P) \mathcal{T}_{P}{ }^{\circ} \mathbf{S}_{i}{ }^{\circ}=2 \delta P\left[\begin{array}{c}
\mathrm{C}_{2 \psi} \mathrm{S}_{2 P} \\
-\mathrm{S}_{2 P} \\
\mathrm{~S}_{2 \psi} \mathrm{C}_{\Delta+Q} \mathrm{C}_{2 P} \\
-\mathrm{S}_{2 \psi} \mathrm{S}_{\Delta+Q} \mathrm{C}_{2 P}
\end{array}\right] \text {, }
$$

which, substituted into Eq. (6), yields

$$
\left[\begin{array}{l}
\delta \Delta \\
\delta \psi
\end{array}\right]=\delta P\left[\begin{array}{c}
0 \\
\mathrm{~S}_{2 \psi} / \mathrm{S}_{2 P}
\end{array}\right] .
$$

$\Delta$ is not shifted at all, and $\delta \psi$ is compensated for if measurements at $P$ and $-P$ are performed and $\psi$ is averaged. For this case of two-zone measurement, we have studied the second-order errors caused by $\delta P$ by using the numerical method. Theoretically, $\delta \psi$ and $\delta \Delta$ should be functions of $P$, $\Delta$, and $\psi$; fortunately, $\Delta$ turns out to have no influence on the errors $\delta \Delta$ and $\delta \psi$ :

$$
\begin{aligned}
& \delta \Delta=\delta \Delta(P, \psi), \\
& \delta \psi=\delta \psi(P, \psi) .
\end{aligned}
$$

In general, we should like to know both $\delta \Delta$ and $\delta \psi$. This, however, requires two graphs. For this reason we introduce $\delta \eta=\left[(\delta \Delta)^{2}+(\delta \psi)^{2}\right]^{1 / 2}$, the largest distance between an actually measured $(\Delta, \psi)$ point and the theoretical one in the $(\Delta$, $\psi$ ) plane. Subsequently we can make a plot of the contours of contrast $\delta \eta$ in the $(P, \psi)$ plane. We have chosen a fixed $\delta P$

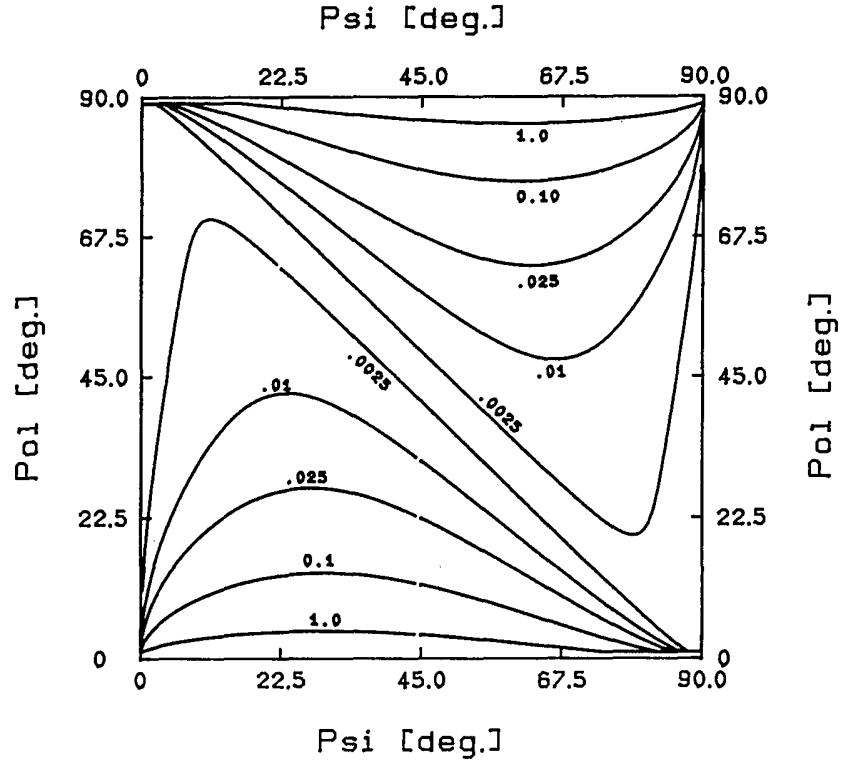

Fig. 2. Errors caused by a polarizer azimuth error $\delta P=1^{\circ}$. If a two-zone measurement is performed, errors $\delta \Delta$ and $\delta \psi$ caused by an azimuth error of the polarizer $\delta P$ are eliminated to first order. This plot shows the remaining higher-order error [expressed as $(\delta \eta)^{2}=$ $\left.(\delta \Delta)^{2}+(\delta \psi)^{2}\right]$ in the $\psi-P$ plane; $\Delta$ has no influence.

$=1^{\circ}$. In Fig. 2, the contours were drawn for $\delta \eta=0.0025^{\circ}$, $0.01^{\circ}, 0.025^{\circ}, 0.10^{\circ}$, and $1^{\circ}$. Figure 2 shows that all errors are eliminated on the diagonal $P=90^{\circ}-\psi$ and on the sides $\psi$ $=0^{\circ}, 90^{\circ}$ the sides of no importance because we already have seen that, in general, errors increase for $\psi$ near $0^{\circ}, 90^{\circ}$. However, if $P$ is chosen between $25^{\circ}$ and $65^{\circ}$, the error is largely eliminated by the two-zone average, and the remaining error is $<0.025^{\circ}$. It should be emphasized that these errors are now dominated by the second-order contribution of $\delta P$, thus exhibiting a $\delta \eta$ proportional to $\delta P^{2}$.

To obtain first-order $(\delta \Delta, \delta \psi)$ for an analyzer azimuth error $\delta A$, we have to deviate a little from our formalism. The light flux $\delta I_{\text {det }}$ becomes

$$
\begin{aligned}
\delta I_{\mathrm{det}} & =\delta_{1} \mathcal{T}_{A} \frac{\mathrm{d} \mathscr{R}}{\mathrm{d} A} \delta A \mathcal{T}_{S}{ }^{\circ} \mathcal{T}_{C}{ }^{\circ} \mathcal{R}^{-1}\left(P^{\circ}\right) \mathcal{T}_{P}{ }^{\circ} \mathbf{S}_{i}{ }^{\circ} \\
& =2 \delta A\left(0, \mathrm{~S}_{2 A}, \mathrm{C}_{2 A}, 0\right) \mathcal{T}_{S}{ }^{\circ} \mathcal{T}_{C}{ }^{\circ} \mathcal{R}^{-1}\left(P^{\circ}\right) \mathcal{T}_{P}{ }^{\circ} \mathbf{S}_{i}{ }^{\circ} .
\end{aligned}
$$

Obviously the vector $\left[\alpha_{0}, \alpha_{c}, \alpha_{s},-\right]$ is found by obtaining the

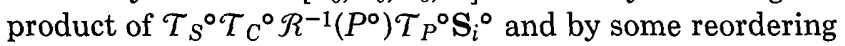
of the result:

$$
\left[\begin{array}{c}
\alpha_{0} \\
\alpha_{c} \\
\alpha_{s} \\
-
\end{array}\right]=2 \delta A\left[\begin{array}{c}
0 \\
\mathrm{~S}_{2 \psi} \mathrm{C}_{\Delta+Q} \mathrm{~S}_{2 P} \\
\mathrm{C}_{2 \psi}-\mathrm{C}_{2 P} \\
-
\end{array}\right]
$$

Substitution of this equation into Eq. (6) yields

$$
\left[\begin{array}{c}
\delta \Delta \\
\delta \psi
\end{array}\right]=\frac{\delta A}{\mathrm{~S}_{2 P} \mathrm{~S}_{2 \psi}}\left[\begin{array}{l}
2\left(\mathrm{C}_{2 P}-\mathrm{C}_{2 \psi}\right) \mathrm{S}_{\Delta+Q} \\
\left(1-\mathrm{C}_{2 P} \mathrm{C}_{2 \psi}\right) \mathrm{S}_{2 \psi} \mathrm{C}_{\Delta+Q}
\end{array}\right],
$$

which again is antisymmetric in $P$. Logically we study the behavior of $\delta \eta$, which is the sum of squares of the remaining errors when a two-zone average is used, as a function of $\Delta, \psi$, 


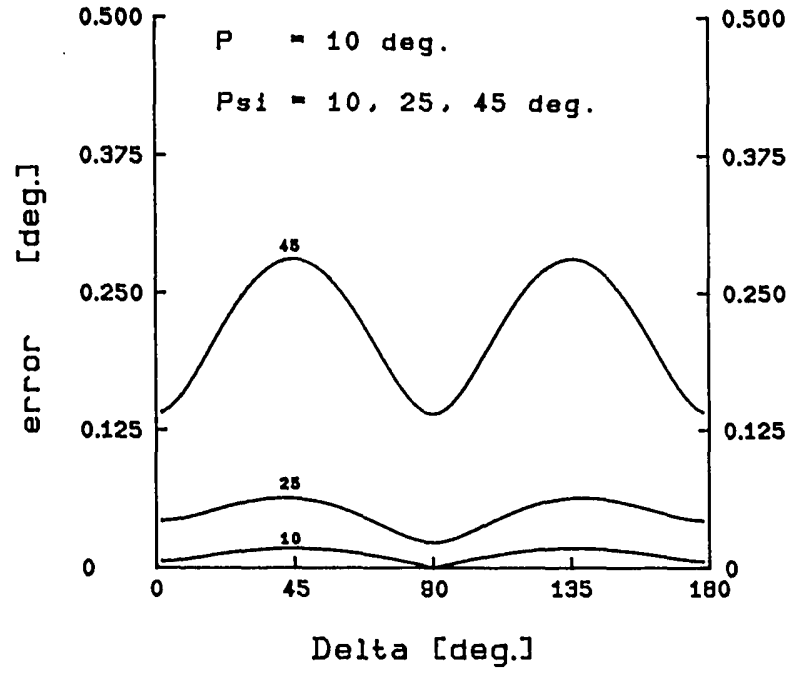

Fig. 3. The error $\delta \eta$ caused by a badly aligned analyzer as a function of $\Delta$ and for some arbitrary values for $\psi$ and $P$. All curves clearly exhibit minima for $\Delta=0^{\circ}, 90^{\circ}$ and maxima for $\Delta=45^{\circ}, 135^{\circ}$.

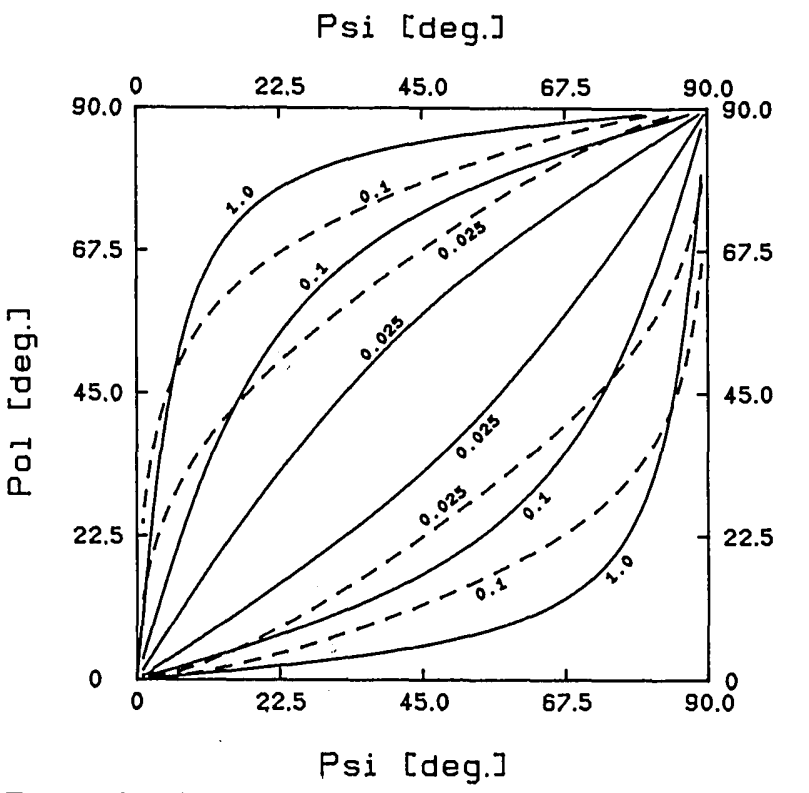

Fig. 4. Second-order errors $\delta \eta$ caused by bad alignment of the analyzer $\left(\delta A=1^{\circ}\right)$. The picture presents the lower and upper bounds for the error $\delta \eta$ in the $\psi-P$ plane, denoted by the dashed and solid lines, respectively.

and $P: \quad \delta \eta=\delta \eta(\Delta, \psi, P)$. We have plotted $\delta \eta$ as a function of $\triangle$ with $P=10^{\circ}, \psi=10^{\circ}, 25^{\circ}, 45^{\circ}$, and $\delta A=1^{\circ}$ in Fig. 3. All curves have a minimum at $\Delta=0^{\circ}, 90^{\circ}$ and a maximum at $\Delta$ $=45^{\circ}$ or $135^{\circ}$. For arbitrary values of $P$ and $\psi$ we always find the minima and maxima thus located, and we may well treat these values as bounds on $\delta \eta$. Figure 4 shows these bounds in the $P-\psi$ plane. The upper bounds are denoted by the solid lines, and the lower bounds are denoted by the dashed ones. The curves for $\delta \eta=0.01$ and $\delta \eta=0.1^{\circ}$ are drawn with $\delta A=1^{\circ}$. From this plot we may conclude that $P$ is safely chosen between $25^{\circ}$ and $65^{\circ}$.

The compensator is the last component whose misalignment certainly has to be studied. Our formalism yields

$$
\begin{aligned}
{\left[\begin{array}{c}
\delta \alpha_{0} \\
\delta \alpha_{c} \\
\delta \alpha_{s} \\
-
\end{array}\right]=\mathcal{T}_{S}^{\circ}\left(\frac{\mathrm{d} \mathcal{R}^{-1}}{\mathrm{~d} C} \mathcal{T}_{C}+\mathcal{T}_{C} \frac{\mathrm{d} \mathscr{R}}{\mathrm{d} C}\right) \delta C \mathscr{R}\left(P^{\circ}\right) \mathcal{T}_{P}{ }^{\circ} \mathbf{S}_{i}^{\circ} } \\
= \\
=\left[\begin{array}{c}
-\left(1-\mathrm{C}_{Q}\right) \mathrm{C}_{2 \psi} \mathrm{S}_{2 P} \\
\left(1-\mathrm{C}_{Q}\right) \mathrm{S}_{2 P} \\
\left(\mathrm{C}_{\Delta}-\mathrm{C}_{\Delta+Q}\right) \mathrm{C}_{2 P} \\
-
\end{array}\right] \delta C .
\end{aligned}
$$

From Eq. (6) we obtain

$$
\left[\begin{array}{l}
\delta \Delta \\
\delta \psi
\end{array}\right]=\frac{\delta C}{\mathrm{~S}_{2 P}}\left[\begin{array}{c}
-2 \mathrm{~S}_{Q} \mathrm{C}_{2 P} \\
\left(1-\mathrm{C}_{Q}\right) \mathrm{S}_{2 \psi}
\end{array}\right]
$$

This error can also be eliminated if a two-zone measurement is performed. Again, we study the second-order error of $\delta \eta$, which is in principle a many-variable function: $\delta \eta=\delta \eta(P, Q$, $\Delta, \psi)$. However, $\Delta$ is of no importance, while the influence of $\psi$ becomes notable for $Q$ values near $180^{\circ}$ only. Figure 5 shows the contours of constant $\delta \eta$ on the remaining $Q-P$ plane for $\delta C=1^{\circ}$. The differences in the line types indicate different $\psi$ values $\left(\psi=30^{\circ}, 45^{\circ}\right)$. Again, we see that $P$ is chosen safely between $25^{\circ}$ and $65^{\circ}$, yielding errors of less than $0.1^{\circ}$ if a two-zone measurement is applied.

\section{Windows}

Birefringence is the main source for window errors, and the window is therefore treated as a small-retardation wave plate. $^{2,6,12}$ In our Taylor formalism the expansion becomes

$$
T_{w}=1+\left[\begin{array}{cccc}
0 & 0 & 0 & 0 \\
0 & 0 & 0 & -\mathrm{S}_{2 \phi w} \\
0 & 0 & 0 & \mathrm{C}_{2 \phi w} \\
0 & -\mathrm{S}_{2 \phi w} & -\mathrm{C}_{2 \phi w} & 0_{0}
\end{array}\right] \delta w,
$$

where $\delta w$ and $\phi w$ correspond to the (small) phase retardation and the azimuth of the fast axis, respectively.

We first consider the entrance window $\mathcal{T}_{w}$. On substitution of Eq. (23) into our formalism, we obtain

$$
\left[\begin{array}{c}
\delta \alpha_{0} \\
\delta \alpha_{c} \\
\delta \alpha_{s} \\
-
\end{array}\right]=\left[\begin{array}{c}
-\mathrm{S}_{Q} \mathrm{C}_{2 \psi} \mathrm{S}_{2 P} \mathrm{~S}_{2 \phi_{w}} \\
\mathrm{~S}_{Q} \mathrm{~S}_{2 P} \mathrm{~S}_{2 \phi_{w}} \\
\left\{\mathrm{~S}_{\Delta} \mathrm{S}_{2 \psi} \mathrm{C}_{2 P} \mathrm{~S}_{2 \phi_{w}}-\mathrm{S}_{\Delta+Q} \mathrm{~S}_{2 \psi} \mathrm{S}_{2 P} \mathrm{C}_{2 \phi_{w}}\right\} \\
-
\end{array}\right] \delta_{w} .
$$

We should note that the compensator $Q$ distinguishes itself from the sample $\Delta$; we are not permitted simply to add them, as in the previous cases. On substitution of Eq. (24) into Eq. (6), we finally obtain

$$
\left[\begin{array}{c}
\delta \Delta \\
\delta \psi
\end{array}\right]=\frac{\delta w}{\mathrm{~S}_{2 P}}\left[\begin{array}{c}
\mathrm{S}_{2 P} \mathrm{C}_{2 \phi_{w}}-\mathrm{C}_{Q} \mathrm{C}_{2 P} \mathrm{~S}_{2 \phi_{w}} \\
1 / 2 \mathrm{~S}_{Q} \mathrm{~S}_{2 \psi} \mathrm{S}_{2 \phi_{w}}
\end{array}\right] .
$$

$\delta \psi$ averages out if a two-zone measurement is applied. Regarding $\delta \Delta$, we notice that part of the error does not cancel. This is expected because if the fast axis of the window is aligned with the plane of incidence of the sample $\left(\phi_{w}=0\right)$, then the sample $\Delta$ and window retardation $\delta w$ should add without even being distinguishable. 


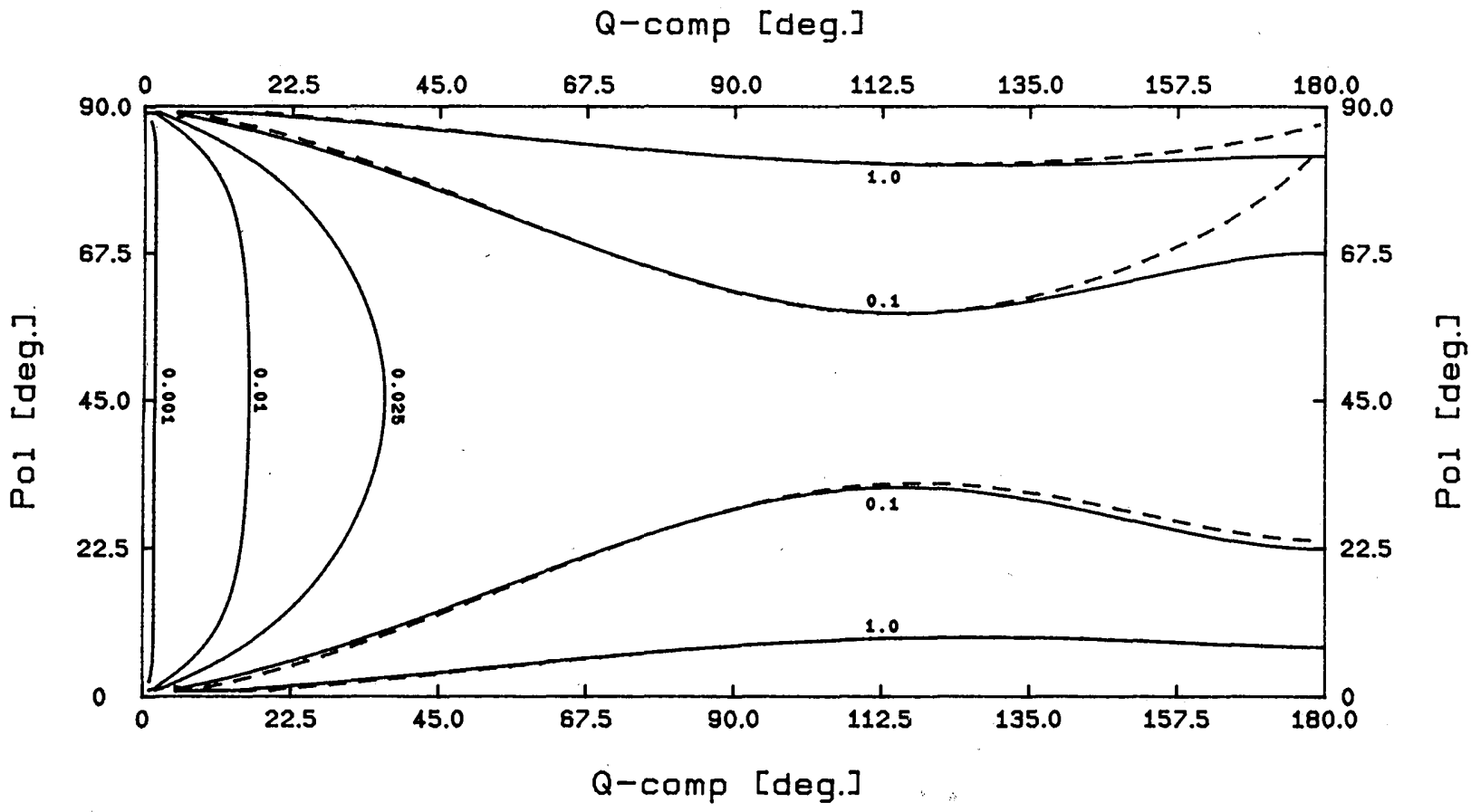

Fig. 5. Second-order errors $\delta \eta$ caused by a misaligned compensator $\left(\delta C=1^{\circ}\right)$. $\delta \eta$ is independent of $\Delta$ and only slightly dependent on $\psi$ and consequently is depicted in the $Q-P$ plane. Solid lines denote the results for $\psi=45^{\circ}$, and dashed lines correspond to $\psi=30^{\circ}$.

The perturbations caused by the exit window $\left(\delta w^{\prime}, \phi w^{\prime}\right)$ become

$$
\left[\begin{array}{c}
\delta \alpha_{0} \\
\delta \alpha_{c} \\
\delta \alpha_{0} \\
-
\end{array}\right]=\mathrm{S}_{\Delta+Q} \mathrm{~S}_{2 P} \mathrm{~S}_{2 \psi}\left[\begin{array}{c}
0 \\
\mathrm{~S}_{2 \phi \omega^{\prime}} \\
-\mathrm{C}_{2 \phi \omega^{\prime}} \\
-
\end{array}\right] \delta w^{\prime},
$$

which yields

$$
\left[\begin{array}{c}
\delta \Delta \\
\delta \psi
\end{array}\right]=\frac{\delta w^{\prime}}{\mathrm{S}_{2 \psi} \mathrm{S}_{2 P}}\left[\begin{array}{c}
\mathrm{C}_{\Delta+Q}\left(\mathrm{C}_{2 \psi}-\mathrm{C}_{2 P}\right) \mathrm{S}_{2 \phi_{w^{\prime}}}+\mathrm{S}_{2 \psi} \mathrm{S}_{2 P} \mathrm{C}_{2 \phi_{w^{\prime}}} \\
1 / 2 \mathrm{~S}_{\Delta+Q} \mathrm{~S}_{2 \psi}\left(1-\mathrm{C}_{2 P} \mathrm{C}_{2 \psi}\right) \mathrm{S}_{2 \phi_{w^{\prime}}}
\end{array}\right] \text {. }
$$

Again $\delta \psi$ and part of $\delta \Delta$ cancel out in the two-zone average. In general we should realize that for samples with small $\mathbf{S}_{\Delta}$ the windows are going to dominate the phase retardation of the system $\mathcal{T}_{w} \mathcal{T}_{s} \mathcal{T}_{w}$, and we actually measure the effective phase retardation of the windows. This situation can be improved by a two-zone measurement, but the error in $\Delta$ can never be completely eliminated because the contributions $\delta w \cos \left(2 \phi_{w}\right)$ of entrance and exit windows are indistinguishable from the $\Delta$ sample.

\section{STATISTICAL ERRORS}

Apart from all systematic errors, we should not neglect the existence of statistical errors. Statistical errors can be attributed to uncorrelated noise (detector noise or shot noise of low-intensity light sources) and to correlated intensity perturbation caused, for example, by sample vibrations or power supplies (the main frequency). Uncorrelated noise was already discussed by Aspnes. ${ }^{14}$ Concerning the correlated perturbations, we present a totally new description.

Uncorrelated perturbations can be discussed only by using their standard deviations. The standard deviations of $\alpha_{0}, \alpha_{c}$, and $\alpha_{s}$ due to noise are interrelated; denoting the standard deviation of $\alpha_{0}, \alpha_{c}$, and $\alpha_{s}$ by $\sigma_{0}, \sigma_{c}$, and $\sigma_{s}$, respectively, Aspnes ${ }^{14}$ derived

$$
\sigma_{c}=\sigma_{s}=\left(2 \sigma_{0}\right)^{1 / 2} .
$$

A quantitative expression for $\sigma_{0}$ is difficult to derive; $\sigma_{0}$ is inversely proportional to the square root of the total acqusition time $(\tau)$ and light intensity:

$$
\sigma_{0} \sim\left(\tau I_{0}\right)^{-1 / 2} .
$$

We should note that we cannot simply use Eq. (6) to relate the standard deviation in $\Delta$ and $\psi\left(\sigma_{\Delta}\right.$ and $\left.\sigma_{\psi}\right)$ to $\sigma_{0}, \sigma_{c}$, and $\sigma_{s}$. The standard deviation $\left(\sigma_{f}\right)$ of a function $f(x, y, \ldots)$ is calculated from

$$
\sigma_{f}^{2}=\left(\frac{\delta f}{\delta x}\right)^{2} \sigma_{x}^{2}+\left(\frac{\delta f}{\delta y}\right)^{2} \sigma_{y}{ }^{2}+\ldots,
$$

where $\sigma_{x}$ and $\sigma_{y}$ denote the standard deviations of the variables $x$ and $y$, respectively. Hence, by squaring all matrix elements of Eq. (5), we obtain a matrix that relates $\sigma_{\Delta}{ }^{2}$ and $\sigma_{\psi}{ }^{2}$ to the squared standard deviations of the normalized Fourier coefficients $\hat{\alpha}_{c}$ and $\hat{\alpha}_{s}, \hat{\sigma}_{c}{ }^{2}$ and $\hat{\sigma}_{s}{ }^{2}$, respectively. A similar matrix relates $\hat{\sigma}_{c}{ }^{2}$ and $\hat{\sigma}_{s}{ }^{2}$ to $\sigma_{c}{ }^{2}$, and $\sigma_{s}{ }^{2}$. From the product of both matrices, we find that

$$
\left[\begin{array}{c}
\sigma_{\Delta}{ }^{2} \\
\sigma_{\psi}{ }^{2}
\end{array}\right]=\frac{1}{\mathrm{~S}_{2 P}{ }^{4} \mathrm{~S}_{2 \psi}{ }^{4} \mathrm{~S}_{\Delta}{ }^{2}}\left[\begin{array}{ccc}
\frac{\left(\mathrm{C}_{2 \psi}-\mathrm{C}_{2 P}\right)^{4}+\mathrm{S}_{2 P}{ }^{4} \mathrm{~S}_{2 \psi}{ }^{4}}{\left(1-\mathrm{C}_{2 P} \mathrm{C}_{2 \psi}\right)^{2}} \mathrm{C}_{\Delta}{ }^{2} & \left(\mathrm{C}_{2 \psi}-\mathrm{C}_{2 \psi}\right)^{2} \mathrm{C}_{\Delta}{ }^{2} & \mathrm{~S}_{2 \psi}{ }^{2} \mathrm{~S}_{2 P}{ }^{2} \\
1 /{ }_{4}\left(\mathrm{C}_{2 \psi}-\mathrm{C}_{2 P}\right)^{2} \mathrm{~S}_{2 \psi}{ }^{2} \mathrm{~S}_{\Delta}{ }^{2} & 1 / 4\left(1-\mathrm{C}_{2 P} \mathrm{C}_{2 \psi}\right)^{2} \mathrm{~S}_{2 \psi}{ }^{2} \mathrm{~S}_{\Delta}{ }^{2} & 0
\end{array}\right]\left[\begin{array}{c}
\sigma_{0}{ }^{2} \\
\sigma_{c}{ }^{2} \\
\sigma_{s}{ }^{2}
\end{array}\right]
$$


After the substitution of Eq. (28) we get the same result as was obtained by Aspnes. ${ }^{14}$ From Eqs. (28)-(30), we could calculate optimum polarizer settings $P$ at which measurements should be performed. However, this subject has been treated extensively by Aspnes, and we direct anyone interested to his work. ${ }^{14}$

The correlated intensity perturbation constitutes another source of statistical errors. Suppose that one uses a light source that shows periodic intensity fluctuations, that the sample or polarizer vibrates, or that the main frequency is picked up somewhere in the electronics. These frequencies may be well known, but their actual phase at the moment the measurement starts is unknown and changes from one measurement to another, thus introducing arbitrary errors. What mainly interests us is which frequencies do cause the major perturbations. Here we present a treatment of a fluctuating light source. All other kinds of fluctuation are assumed to perturb $\alpha_{0}, \alpha_{c}$, and $\alpha_{s}$ in a similar way.

Now let us assume that the light source produces the light flux $I(t)$ :

$$
I(t)=I^{\circ}+\delta I(\omega) \cos (\omega t+\phi),
$$

where we have introduced an arbitrary phase factor $\phi$ at the moment the measurement is started. The transmitted light flux becomes

$$
\begin{aligned}
I_{\mathrm{det}}(t)= & {\left[I^{\circ}+\delta I(\omega) \cos (\omega t+\phi)\right]\left[1-\mathrm{C}_{2 P} \mathrm{C}_{2 \psi}\right.} \\
& \left.+\left(\mathrm{C}_{2 p}-\mathrm{C}_{2 \psi}\right) \cos 2 \omega_{A} t+\mathrm{S}_{2 P} \mathrm{~S}_{2 \psi} \mathrm{C}_{\Delta} \sin 2 \omega_{A} t\right] .
\end{aligned}
$$

The coefficients $\alpha_{0}, \alpha_{c}$, and $\alpha_{s}$ are retrieved from $I_{\text {det }}$ by using Eqs. (3a) and (3b). Applying this formalism to Eq. (32), performing the integrals, and retaining positive frequencies $\omega$, we obtain

$$
\begin{aligned}
\alpha_{0}= & \left(1-\mathrm{C}_{2 P} \mathrm{C}_{2 \psi}\right) I^{\circ}+\left(1-\mathrm{C}_{2 P} \mathrm{C}_{2 \psi}\right) \delta I(\omega) \operatorname{sinc}(\omega \tau ; \phi) \\
& \times\left(\mathrm{C}_{2 P}-\mathrm{C}_{2 \psi}\right) \delta I(\omega) \operatorname{sinc}\left[\left(2 \omega_{A}-\omega\right) \tau ; \phi\right] \\
& \times \mathrm{S}_{2 P} \mathrm{~S}_{2 \psi} \mathrm{C}_{\Delta} \delta I(\omega) \operatorname{sinc}\left[\left(2 \omega_{A}-\omega\right) \tau ; \phi+\frac{\pi}{2}\right], \\
\alpha_{c}= & \left(\mathrm{C}_{2 P}-\mathrm{C}_{2 \psi}\right) I^{\circ}+\left(1-\mathrm{C}_{2 P} \mathrm{C}_{2 \psi}\right) \delta I(\omega) \operatorname{sinc}\left[\left(2 \omega_{A}-\omega\right) \tau ; \phi\right] \\
& \times\left(\mathrm{C}_{2 P}-\mathrm{C}_{2 \psi}\right) \delta I(\omega)\left\{\operatorname{sinc}(\omega \tau ; \phi)+\operatorname{sinc}\left[\left(4 \omega_{A}-\omega\right) \tau ; \phi\right]\right\} \\
& \times \mathrm{S}_{2 P} \mathrm{~S}_{2 \psi} \mathrm{C}_{\Delta} \delta I(\omega) \operatorname{sinc}\left[\left(4 \omega_{A}-\omega\right) \tau ; \phi+\frac{\pi}{2}\right], \\
\alpha_{s}= & \mathrm{S}_{2 p} \mathrm{~S}_{2 \psi} \mathrm{C}_{\Delta} I^{\circ}+\left(1-\mathrm{C}_{2 P} \mathrm{C}_{2 \psi}\right) \delta I(\omega) \operatorname{sinc}\left[\left(2 \omega_{A}-\omega\right) \tau ; \phi+\frac{\pi}{2}\right] \\
& \times\left(\mathrm{C}_{2 P}-\mathrm{C}_{2 \psi}\right) \delta I(\omega) \operatorname{sinc}\left[\left(4 \omega_{A}-\omega\right) \tau ; \phi+\frac{\pi}{2}\right] \\
& \times \mathrm{S}_{2 P} \mathrm{~S}_{2 \psi} \mathrm{C}_{\Delta} \delta I(\omega)\left\{\operatorname{sinc}\left[\left(4 \omega_{A}-\omega\right) \tau ; \phi\right]+\operatorname{sinc}(\omega \tau ; \phi)\right\} .
\end{aligned}
$$

$\tau$ is twice an integer number of analyzer periods $(\tau=4 \pi N /$ $\left.\omega_{A}\right)$, and $\operatorname{sinc}(x ; \phi)$ is a generalization of the sinc function ${ }^{15}$

$$
\operatorname{sinc}(x ; \phi)=\frac{\sin (x+\phi)-\sin \phi}{x} .
$$

In Fig. 6 we have plotted this generalized sinc function. First, note that all coefficients $\alpha_{0}, \alpha_{c}$, and $\alpha_{s}$ are mixed. The amount of mixing is related to the quotient $\delta I(\omega) / I^{\circ}$, and obviously all fluctuations $\delta I(\omega)$ have to be strongly suppressed. By studying $\operatorname{sinc}(x ; \phi)$, we can obtain more precise

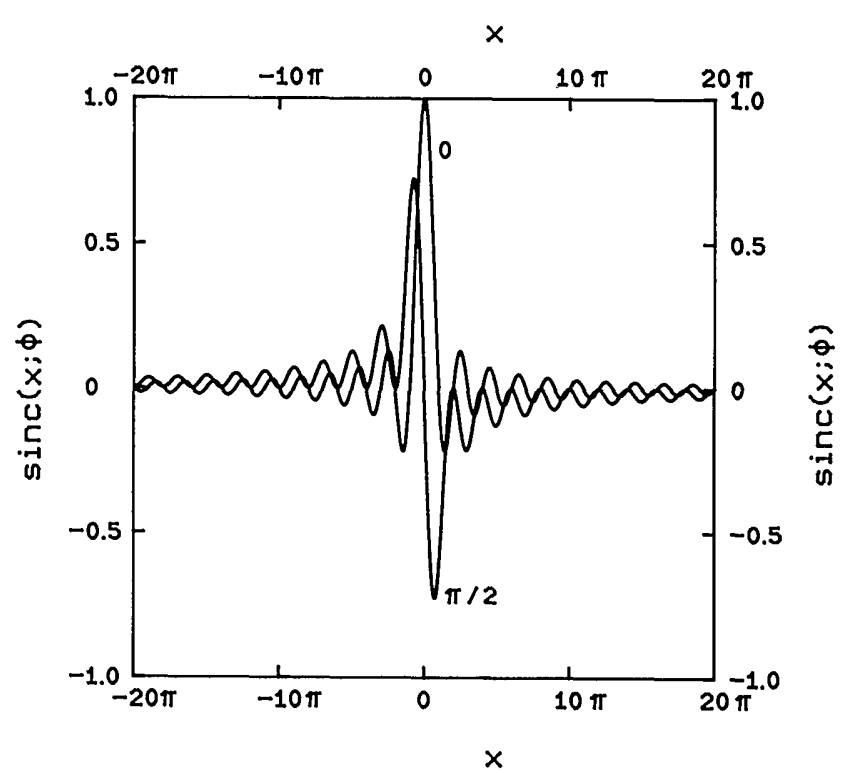

Fig. 6. The generalized $\operatorname{sinc}(x ; \phi)$ function as defined by Eq. (34) for $\phi=0$ and $\phi=\pi / 2 \mathrm{rad}$. The figure clearly shows the localized and bounded character of the function.

conclusions. Our generalized sinc function is bounded and strongly located near $x=0$, as is shown in Fig. 6. We may neglect any contribution for $x<-8 \pi$ and $x>8 \pi$ :

$$
\operatorname{sinc}(x, \phi) \approx 0 \quad \text { if }|x|>8 \pi .
$$

Considering this localized and bounded character of $\operatorname{sinc}(x$, $\phi)$, which still is completely arbitrary because of the phase factor $\phi$, we can conclude that the only frequency regions involved are

$$
\begin{gathered}
0<\omega<\frac{2 \omega_{A}}{N}, \\
2 \omega_{A}-\frac{2 \omega_{A}}{N}<\omega<2 \omega_{A}+\frac{2 \omega_{A}}{N}, \\
4 \omega_{A}-\frac{2 \omega_{A}}{N}<\omega<4 \omega_{A}+\frac{2 \omega_{A}}{N},
\end{gathered}
$$

Obviously these regions narrow with the increasing number of cycles; however, it should be emphasized that only the regions narrow and that possible errors, caused by a periodic perturbation within a region, are not affected. Such perturbations have to be avoided in all cases.

In principle we can substitute the deviations $\delta \alpha_{0}, \delta \alpha_{c}$, and $\delta \alpha_{s}$ obtained by Eqs. (33) into Eq. (6). However, we would arrive at a complicated expression. Generally, it will be sufficient to realize that the errors become dominant mainly if $\mathrm{S}_{\Delta} \approx 0$ or $P, \psi \approx 0$ or if the perturbation is localized near $2 \omega_{A}$ or $4 \omega_{A}$. Slow variation of the light intensity $(\omega \approx 0)$ may be neglected because these perturbations do not cause a mixing of the coefficients $\alpha_{0}, \alpha_{c}$, and $\alpha_{s}$, as can be seen from Eqs. (33).

\section{DISCUSSION}

In this paper we tried to obtain a better understanding of the main phenomena causing errors in RAE. We have consid- 
ered two kinds of errors: systematic errors and statistical errors. In general we conclude that many errors do distort the single-zone measurement, and absolute accuracy is not obtained, especially if the ellipsometer is applied at small $P$ or if $\mathrm{S}_{\Delta}$ or $\mathrm{S}_{2 \psi}$ becomes small. In all these cases we should expect errors in $\delta \Delta$ and $\delta \psi$ of the order of degrees. This situation is much improved when a two-zone measurement is applied; in this case, with the exception of window errors, all first-order errors are eliminated. Apparently errors are more easily eliminated in the RAE than in the traditional nulling ellipsometer, which requires a four-zone measurement to do so. ${ }^{2}$

One type of error, the azimuth error, directly emerges from the calibration. We often see problems in this calibration, for instance, for $\mathrm{S}_{\Delta} \approx 0$. The residuals do not exhibit a pronounced minimum, thus obstructing the determination of the plane of incidence. We should like to know how accurately the plane of incidence has to be known if we are to obtain good results. Figures 2, 4, and 5 show that when $P$ is chosen properly and a two-zone measurement is applied these errors will be less than $0.1^{\circ}$ with $\delta P, \delta A$, and $\delta C=1^{\circ}$. Certainly these errors are much less than the errors caused by windows, which introduce the main errors.

We emphasize that a badly chosen analyzer frequency $\omega_{A}$ can increase statistical errors strongly. $\omega_{A}$ should be chosen such that any periodic perturbation $(\omega)$ does not lie in the regions near $2 \omega_{A}$ and $4 \omega_{A}$.

\section{REFERENCES}

1. R. M. A. Azzam and N. M. Bashara, "Ellipsometry with imperfect components including incoherent effects," J. Opt. Soc. Am. 61, 1380-1391 (1971).
2. R. M. A. Azzam and N. M. Bashara, "Unified analysis of ellipsometry errors due to imperfect components, cell-window birefringence, and incorrect azimuth angles," J. Opt. Soc. Am. 61, 600-607 (1971).

3. R. M. A. Azzam and N. M. Bashara, "General treatment of the effect of cell windows in ellipsometry," J. Opt. Soc. Am. 61, 773776 (1971).

4. R. M. A. Azzam and N. M. Bashara, Ellipsometry and Polarized Light (North-Holland, Amsterdam, 1977).

5. D. E. Aspnes, "Measurement and correction of first-order errors in ellipsometry," J. Opt. Soc. Am. 61, 1077-1085 (1971).

6. A. Straaijer, L. J. Hanekamp, and G. A. Bootsma, "The influence of cell window imperfections on the calibration and measured data of two types of rotating-analyzer ellipsometers," Surf. Sci. 96, 217-231 (1980).

7. F. L. McCrackin, "Analyses and corrections of instrumental errors in ellipsometry," J. Opt. Soc. Am. 60, 57-63 (1970).

8. R. H. Muller, "Present status of automatic ellipsometers," Surf. Sci. 56, 19-36 (1976).

9. P. S. Hauge, "Generalized rotating compensator ellipsometry," Surf. Sci. 56, 148-160 (1976).

10. D. E. Aspnes and A. A. Studna, "High precision scanning ellipsometer," Appl. Opt. 14, 220-228 (1975).

11. Y. I. van der Meulen and N. C. Hien, "Design and operation of an automated, high-temperature ellipsometer," J. Opt. Soc. Am. 64, 804-811 (1974).

12. R. M. A. Azzam and N. M. Bashara, "Analysis of systematic errors in rotating-analyzer ellipsometers," J. Opt. Soc. Am. 64, 1459-1469 (1974)

13. D. E. Aspnes, "Optimizing precision of rotating-analyzer ellipsometers," J. Opt. Soc. Am. 64, 639-646 (1974).

14. D. E. Aspnes, "Effects of component optical activity in data reduction and calibration of rotating-analyzer ellipsometers," J. Opt. Soc. Am. 64, 812-819 (1974).

15. G. A. Korr and T. M. Kom, Mathematical Handbook for Scientists and Engineers (McGraw-Hill, New York, 1968). 